\title{
Cross-sectional quantitative analysis of the natural history of TUBA1A and TUBB2B tubulinopathies
}

\author{
Julian Schröter, MD® ${ }^{1}$, Jan H. Döring, MD¹, Sven F. Garbade, PhD², Georg F. Hoffmann, MD², \\ Stefan Kölker, MD², Markus Ries, MD, PhD $\mathbb{1}^{2}$ and Steffen Syrbe, MD ${ }^{1}{ }^{1}$
}

Purpose: TUBA1A and TUBB2B tubulinopathies are rare neurodevelopmental disorders characterized by cortical and extracortical malformations and heterogenic phenotypes. There is a need for quantitative clinical endpoints that will be beneficial for future diagnostic and therapeutic trials.

Methods: Quantitative natural history modeling of individuals with TUBA1A and TUBB2B tubulinopathies from clinical reports and database entries of DECIPHER and ClinVar. Main outcome measures were age at disease onset, survival, and diagnostic delay. Phenotypical, neuroradiological, and histopathological features were descriptively illustrated.

Results: Mean age at disease onset was 4 (TUBA1A) and 6 months (TUBB2B), respectively. Mortality was equally estimated with $7 \%$ at 3.2 (TUBA1A) and 8.0 years (TUBB2B). Diagnostic delay was significantly higher in $T U B B 2 B$ (12.3 years) compared with TUBA1A tubulinopathy (4.2 years). We delineated the isotype-dependent clinical, neuroradiological, and histopathological phenotype of affected individuals and present brain malformations associated with epilepsy and an unfavorable course of disease.

Conclusion: The natural history of tubulinopathies is defined by the genotype and associated brain malformations. Defined data on estimated survival, diagnostic delay, and disease characteristics of TUBA1A and TUBB2B tubulinopathy will help to raise disease awareness and encourage future clinical trials to optimize genetic testing, family counseling, and supportive care.

Genetics in Medicine (2021) 23:516-523; https://doi.org/10.1038/s41436020-01001-z

Keywords: TUBA1A; TUBB2B; tubulinopathy; natural history; malformations of cortical development

show severe MCDs, i.e., microlissencephaly, lissencephaly with predominant agyria, or diffuse polymicrogyria-like cortical dysplasia, TUBB2B-associated tubulinopathies are mainly characterized by focal (perisylvian) or generalized polymicrogyria-like cortical dysplasia., ${ }^{4,16}$ Another distinctive feature, congenital fibrosis of the extraocular muscles (CFEOM), an ocular motility disorder that has been associated with $T U B B 3$, was observed in one family harboring a $T U B B 2 B$ variant. ${ }^{17,18}$ In both TUBA1A- and TUBB2B-associated tubulinopathies, abnormalities of cerebellar hemispheres and vermis, basal ganglia, and commissural structures are commonly observed. ${ }^{4,16,19}$ Affected patients most frequently show a range of neurodevelopmental disorders including cognitive and motor impairment, abnormal muscular tone, and epilepsy resulting in a high burden of disease. ${ }^{4,5,20}$ Orphanet estimates a prevalence of $<1: 1,000,000$ (tubulinopathy-associated dysgyria; Orpha: 467166). ${ }^{21}$ Previous studies focused on clinical, neuroradiological, and genetic features of tubulinopathies. So far, quantitative natural history data on survival rates, disease onset, and diagnostic delay are scarce but crucial for counseling of afflicted families and for design of future clinical trials with targeted therapeutic approaches. Raising disease awareness by

${ }^{1}$ Division of Pediatric Epileptology, Center for Pediatrics and Adolescent Medicine, University Hospital Heidelberg, Heidelberg, Germany; ${ }^{2}$ Division of Neuropediatrics and Inherited Metabolic Diseases, Center for Pediatrics and Adolescent Medicine, University Hospital Heidelberg, Heidelberg, Germany. Correspondence:

Steffen Syrbe (Steffen.Syrbe@med.uni-heidelberg.de)

These authors contributed equally: Markus Ries, Steffen Syrbe

Submitted 21 July 2020; revised 25 September 2020; accepted: 28 September 2020

Published online: 21 October 2020 
quantitative natural history data has the potential to shorten diagnostic delay and ensure optimal supportive patient care. ${ }^{22}$

In this study, we therefore focused on quantification of relevant clinical endpoints such as survival, disease onset, and diagnostic delay. In addition, we describe cardinal features at disease onset and characterize associated epilepsies to improve the understanding of the clinical course and to complement therapeutic concepts for affected individuals.

\section{MATERIALS AND METHODS}

This study was conducted in compliance with STrengthening the Reporting of OBservational studies in Epidemiology (STROBE) criteria. $^{23}$

\section{Literature review and definition of variables}

We performed a comprehensive literature research on PubMed using the keywords "TUBA1A," "TUBB2B," and "tubulinopathy." Selected reports were published between January 2007 and July 2019. Reports were checked for duplicate mentions and verified by listed variants in the Human Gene Mutation Database at the University of Cardiff (http://www.hgmd.cf.ac.uk/ac/gene.php?gene=TUBA1A/

TUBB2B). We additionally included entries from the databases ClinVar ${ }^{24}$ and DECIPHER ${ }^{25}$ providing clinical information on TUBA1A and $T U B B 2 B$ missense variants depicted as "pathogenic" or "likely pathogenic." Variants without corresponding clinical information were excluded from further analysis. According to the Human Genome Variation Society (HGVS) recommendations, TUBA1A and TUBB2B variants were standardized to the NM_006009.3 and NM_178012.4 transcript of the GRCh37/hg19 human reference genome, respectively, using the Mutalyzer Nomenclature Checker web tool. ${ }^{26}$ Cut-off date for data analysis was 1 July 2019. The following variables were extracted: age and symptoms at disease onset, cardinal symptoms leading to diagnosis, age at diagnosis, last reported age, information on whether the patient is alive or deceased, gestational age at birth or termination of pregnancy (TOP), age at seizure onset, main seizure types, applied antiepileptic drugs (AEDs), neuroradiological and histopathological findings, year of publication, and origin of the patient. If not explicitly stated in the report, the country of the patient's origin has been attributed to the country of the first author's institutional affiliation in the corresponding case description. If temporal information was expressed in semiquantitive terms, we used the following definitions: "at birth" = day 1, "newborn period" = 1 month, "infancy" = 1 year. If the individual's decease was not explicitly stated, individuals were considered alive at the last reported age. In statistical analysis, the term "dead" is used for individuals who died due to TOP in utero or disease complications during lifetime, and was stratified as "severe course of disease" in association analysis. In detail, the main category "symptoms at disease onset" comprised eight major clinical signs. The main category "clinical features" was grouped in subcategories as follows: "epilepsy," "microcephaly," "developmental delay," "motor impairment," “abnormal muscular tone," "further neurological features," "musculoskeletal features," "ocular features" including ocular motility disorders, and "facial dysmorphisms." Cortical and extracortical findings derived from neuroradiological analyses via brain ultrasound and/or magnetic resonance image (MRI) as well as neuropathological analyses delineated in clinical reports and database entries were extracted, allocated to the affected brain region, and categorized by known tubulinopathy-associated, cortical and extracortical malformations comprising 12 and 26 features, respectively. Cohorts were subdivided whether individuals were reported as alive, deceased, or fetus after TOP. Severity stratification of the clinical variable "motor impairment" was oriented to the revised Gross Motor Function Classification System (GMFCS) ${ }^{27}$ as follows: none (no limitations), mild (GMFCS I), moderate (GMFCS II-III), and severe (GMFCS IV-V).

\section{Statistical analysis}

Techniques of descriptive statistics were applied as previously reported. $^{28,29}$ Quantitative variables are illustrated by sample size, mean, and standard deviation. Statistical comparisons between means of the two cohorts were performed with Welch's two-sample $t$-test. A paired $t$-test was used to compare head sizes at birth and at last follow-up of the same individual. Frequencies of descriptive variables are depicted with number, sample size, and percentages. Statistical comparisons of the frequency of descriptive variables between the two cohorts were conducted using the two-tailed Fisher's exact test. Survival was assessed and defined as the time interval between birth and age at death. Data were censored at the time of the last follow-up if the individual was still alive at last follow-up according to the extracted data of the corresponding publication. Survival estimations were calculated using the Kaplan-Meier method. The log-rank test was applied to compare potential differences between subgroups. Diagnostic delay was calculated as the time interval between age at diagnosis and age at disease onset. Prevalence of clinical characteristics was compared between both tubulinopathies. All analyses were performed using R. ${ }^{30}$ The world maps were plotted using the R extension ggmap. ${ }^{31}$ Missing data were not imputed. Sensitivity analyses were not conducted. $P$ values reported were two-sided, with $P \leq 0.05$ considered statistically significant.

\section{Ethics statement}

This study did not make use of individual patient data. All patients had been pseudonymized prior to inclusion into our study.

\section{RESULTS}

In 63 published clinical reports $\left(N_{\mathrm{TUBA} 1 \mathrm{~A}}=49 ; N_{\mathrm{TUBB} 2 \mathrm{~B}}=\right.$ $14)$ and 61 database entries $\left(N_{\text {TUBA1A }}=52 ; N_{\text {TUBв2B }}=9\right)$, we identified 127 TUBA $1 A$ and 43 TUBB2B variants from 190 and 55 individuals, respectively. Forty-two individuals had to be excluded from statistical analysis due to missing clinical data, resulting in an overall study cohort of 203 individuals 
$\left(N_{\text {TUBA } 1 \mathrm{~A}}=155 ; N_{\text {TUBв2B }}=48\right)$. A flow chart delineating the exclusion process is provided in Fig. S1.

\section{Characteristics of the study cohorts}

From 144 reported cases, 120 individuals (83.3\%) with TUBA1A tubulinopathy were born at term (gestational week [GW] 40). Termination of pregnancy (TOP) was performed in 24 cases (16.7\%) around GW 28 (range: GW 21-38) due to severe brain abnormalities on prenatal ultrasound (US) and/ or magnetic resonance imaging (MRI). From 47 cases with TUBB2B tubulinopathy, 40 individuals $(85.1 \%)$ were delivered at term and TOP was induced in 7 cases $(14.9 \%)$ on average in GW 28 (range: GW 16-33). Sexual distribution was balanced in both study cohorts. Details of the study cohorts are illustrated in Table S1. The country of birth is shown in Fig. S2, suggesting a predominant distribution in Western developed countries. Values from descriptive statistics are outlined in Table S2.

\section{Survival estimations}

In the TUBA1A study cohort, five individuals (3.5\%) were deceased at the time of publication (Table S1). Estimated survival analysis using the Kaplan-Meier method showed that $93.4 \%$ of individuals with TUBA1A tubulinopathy were still alive at the age of 3.2 years (Fig. 1). In the TUBB2B study cohort, two individuals (4.3\%) had died during follow-up and $93.3 \%$ of the individuals were alive at the age of 8.0 years (Fig. 1). Survival data showed no significant differences between both study cohorts. One individual of the TUBA1A cohort had died of respiratory failure at 23 months. In the $T U B B 2 B$ cohort, one individual

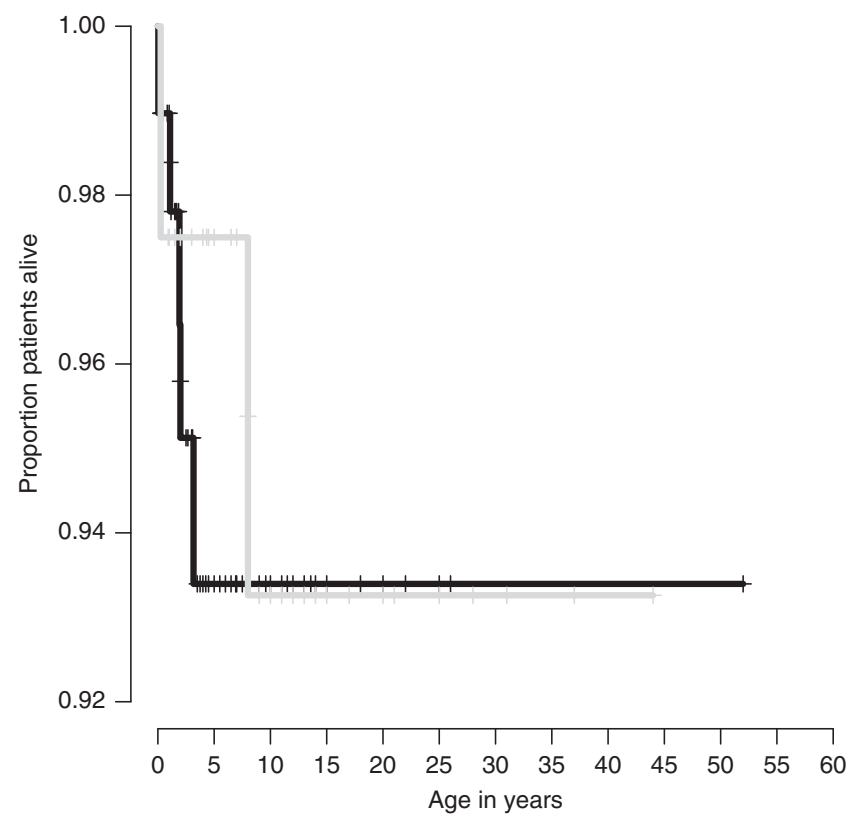

Fig. 1 Age of onset and age at diagnosis of TUBA1A (a) and TUBB2B (b) tubulinopathy. Estimated overall survival distribution for patients with TUBA1A ( $N=97$, black) and TUBB2B tubulinopathies ( $N=40$, gray). Censored individuals are marked with $a+$. Log-rank test, $p=0.8$. deceased at the age of 8 years after recurrent respiratory infections. The cause of death was not further delineated in the remaining cases.

\section{Quantitative and qualitative characterization of the disease onset}

We quantitatively analyzed the age at disease onset in both cohorts. In the TUBA1A cohort, the mean age at disease onset was 4.0 months ( $\mathrm{SD} \pm 5.3$ months; $N=29$ ), which did not differ significantly from the $T U B B 2 B$ cohort (5.9 \pm 8.2 months; $N=17$; Table S2). In the TUBA1A cohort, genetic diagnosis was established at the mean age of 4.5 years $(\mathrm{SD} \pm 4.0$ years; $N=29$ ) whereas the age at diagnosis was significantly higher in the $T U B B 2 B$ cohort $(12.8 \pm 9.5$ years; $N=17 ; P<0.001$; Table S2). Correspondingly, mean diagnostic delay after appearance of initial symptoms differed significantly between the TUBA1A ( $4.2 \pm 4.0$ years) and the TUBB2B cohort $(12.3 \pm$ 9.9 years; $P=0.004$; Fig. 2). Early postnatal symptoms in individuals with TUBA1A variants were most frequently microcephaly (54.3\%), seizures (42.9\%), and muscular hypotonia (37.1\%; Table 1). Most common, partially overlapping symptoms at disease onset in the TUBB2B cohort were developmental delay (47.4\%), seizures $(36.8 \%)$, and muscular hypotonia (21.1\%). Microcephaly (54.3\% vs. $15.8 \%$ ) and facial dysmorphisms (31.4\% vs. $0.0 \%)$ were significantly more prevalent in individuals with TUBA1A tubulinopathy ( $P=0.009$ and $P=0.005$, respectively). Further symptoms at disease onset are shown in Table $\mathbf{1 .}$

\section{Clinical features in the course of disease}

TUBA1A- and TUBB2B-associated disease features are comprehensively outlined in Table 2 and Table S3. During the course of disease, motor impairment, developmental delay, abnormalities of the muscular tone, microcephaly, and epilepsy were the most frequent clinical features but differed in distribution between individuals with TUBA1A and TUBB2B tubulinopathy. Gross motor function was more commonly affected in the TUBA1A (97.3\%) than in the TUBB2B cohort $(73.1 \% ; P=0.001)$ whereas normal motor function was significantly more prevalent in the TUBB2B cohort $(19.2 \%$ vs. $2.9 \%$; $P=0.012)$. Mild, moderate, and severe motor impairment was equally distributed in both cohorts. All deceased patients in the TUBA1A cohort had severe motor impairment. Accordingly, abnormalities of the muscular tone such as spastic di- or tetraplegia, muscular hypo- and dystonia occurred more frequently in the TUBA1A cohort $(89.6 \%$ vs. $48.0 \%$; $P<0.001)$. Occurrence of musculoskeletal comorbidities was likewise distributed in both cohorts $(11.9 \%$ vs. $35.3 \%$; $P=0.061)$. The vast majority of individuals showed delay in cognitive, speech, and motor development. In most cases, more than one developmental area was affected. In the TUBA1A cohort, delay of global development $(95.7 \%$ vs. $76.7 \% ; P=0.005)$, speech $(98.9 \%$ vs. $88.6 \% ; P=0.020$ ), and motor development $(97.8 \%$ vs. $83.8 \%$; $P=0.007)$ were significantly more common than in the $T U B B 2 B$ cohort where mild cognitive disability such as 

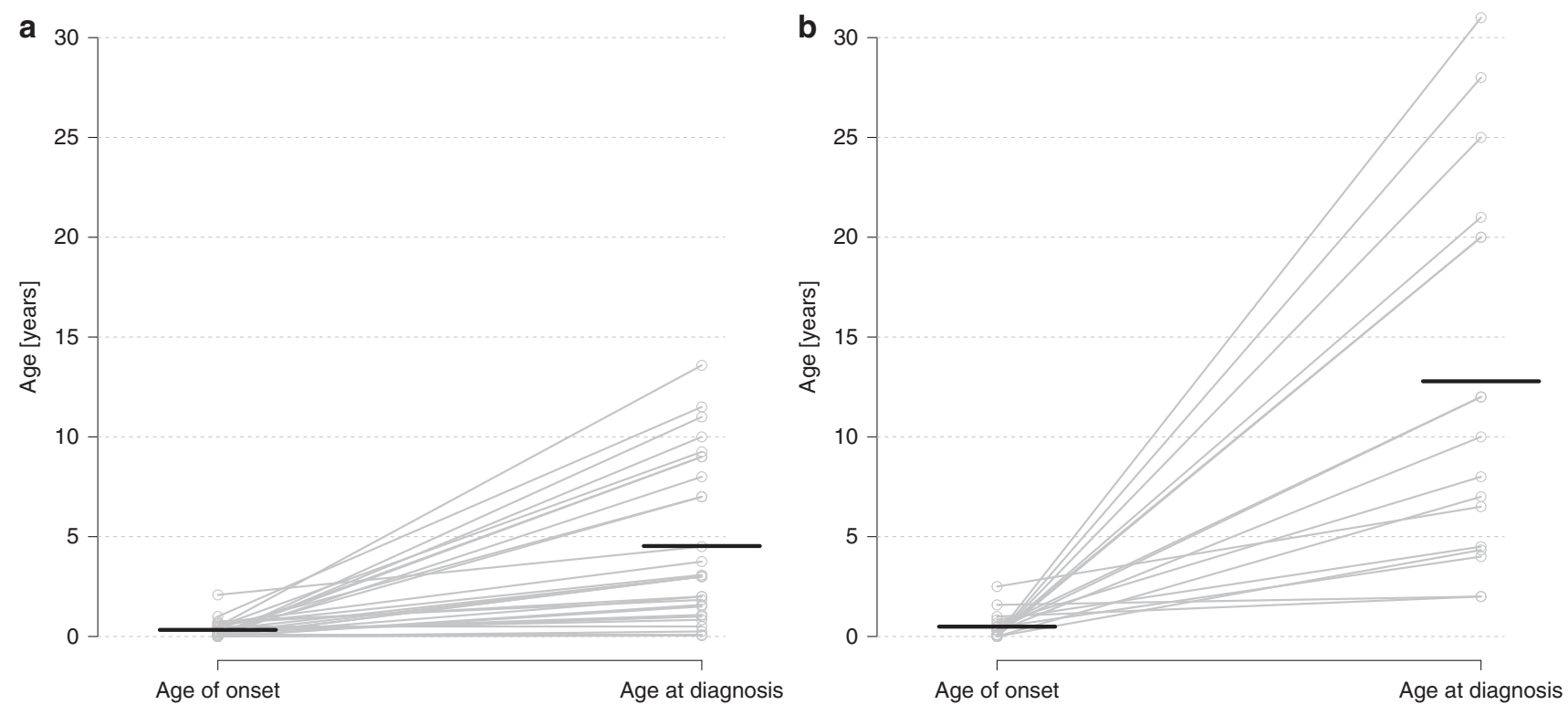

Fig. 2 Age of onset and age at diagnosis of TUBA1A (a) and TUBB2B (b) tubulinopathy. Data were available for $N_{\text {TUBA1A }}=29$ and $N_{\text {TUBB2B }}=17$ individuals, respectively. Horizontal lines indicate the mean. The slopes of connecting lines represent the diagnostic delay between onset of the disease and the time of diagnosis.

Table 1 Symptoms at disease onset in the study cohorts.

\begin{tabular}{llll} 
Symptoms at disease onset & TUBA1A & TUBB2B & $P$ value \\
\hline Microcephaly & $19 / 35$ & $3 / 19$ & $0.009^{\mathrm{a}}$ \\
& $54.3 \%$ & $15.8 \%$ & \\
Facial dysmorphism & $11 / 35$ & $0 / 19$ & $0.005^{\mathrm{a}}$ \\
& $31.4 \%$ & $0.0 \%$ & \\
\hline Developmental delay & $10 / 35$ & $9 / 19$ & 0.234 \\
& $28.6 \%$ & $47.4 \%$ & \\
\hline Muscular hypotonia & $13 / 35$ & $4 / 19$ & 0.358 \\
& $37.1 \%$ & $21.1 \%$ & \\
\hline Seizures & $15 / 35$ & $7 / 19$ & 0.775 \\
& $42.9 \%$ & $36.8 \%$ & \\
\hline Feeding difficulties & $5 / 35$ & $0 / 19$ & 0.149 \\
& $14.3 \%$ & $0.0 \%$ & \\
\hline Poor visual contact & $3 / 35$ & $1 / 19$ & 1.0 \\
& $8.6 \%$ & $5.3 \%$ & \\
\hline Nystagmus & $2 / 35$ & $0 / 19$ & 0.535 \\
& $5.7 \%$ & $0.0 \%$ & \\
\hline Other & $4 / 35$ & $4 / 19$ & 0.431 \\
\hline
\end{tabular}

Proportions are illustrated in numbers (top) and percentages (bottom). ${ }^{\mathrm{a} S i g n i f i c a n t ~ d i f f e r e n c e . ~}$

isolated learning difficulties were more prominent $(1.1 \%$ vs. $14.3 \% ; P=0.006)$. Microcephaly had a similar prevalence in both cohorts $(74.3 \%$ vs. $67.4 \%)$. In contrast, primary microcephaly occurred more frequently in the TUBA1A (88.2\%) than in the TUBB2B cohort $(50.0 \% ; P=0.012)$ and reduction of the occipitofrontal diameter (OFD) was pronounced in both groups at last follow-up $(-3.7$ and $-3.5 \mathrm{SD}$, respectively). Microcephaly in TUBA1A tubulinopathy was progressive from $-2.0 \mathrm{SD}$ at birth compared with
$-4.1 \mathrm{SD}$ at last follow-up in cases with both reported variables $(N=9 ; P=0.004)$. In the TUBB2B cohort, data on OFD at birth was only available in one case. Epilepsy was common in both cohorts: $65.9 \%$ (TUBA1A) and 54.8\% (TUBB2B) of individuals developed seizures during the observation period. The mean age of seizure onset was 7.5 months $\left(N_{\text {TUBAIA }}=\right.$ $25)$ and 33.1 months $\left(N_{\text {TUBв2B }}=14 ; P=0.331\right.$; Table S2). In both cohorts, infantile onset of epilepsy was seen in the majority of cases $(84.6 \%$ vs. $78.6 \%)$ indicating a broader range of age at seizure onset in TUBB2B cases. Infantile spasms were the most frequent seizure type in both cohorts $(32.5 \%$ vs. $27.3 \%$ ). Individuals generally showed a broad range of focal and generalized semiologies that occurred similarly in TUBA1A- and TUBB2B-associated epilepsy. Epilepsy was more often refractory to treatment in the TUBA1A (64.7\%) than in the TUBB2B cohort $(37.5 \%, P=0.235)$. Valproic acid, phenobarbital, levetiracetam, carbamazepine, and topiramate were the most frequently used antiepileptic drugs (AEDs). Further clinical features were facial diplegia (21.7\%), which was exclusively observed in the TUBA1A cohort, and ocular abnormalities, which were described in 58.6\% (TUBA1A) and $65.2 \%(T U B B 2 B)$ of the individuals, respectively. Strabismus and nystagmus were the most common ocular motility disorders. Congenital fibrosis of the extraocular muscles (CFEOM) was exclusively depicted in the TUBB2B cohort. Facial dysmorphisms were generally found in both cohorts but were more commonly reported in TUBA1A $(68.8 \%)$ than in TUBB2B fetuses $(20.0 \%)$ and primarily included retrognathism (19.0\%) and hypertelorism (11.4\%), which were not present in the TUBB2B cohort where individuals primarily showed downturned corners of the mouth $(0.0 \%$ vs. $33.3 \%)$. Reported facial dysmorphisms are delineated in Table S4. 
Table 2 Classification and outcome of epilepsy in the study cohorts.

\begin{tabular}{|c|c|c|c|}
\hline & TUBA1A & $T U B B 2 B$ & $P$ Value \\
\hline \multicolumn{4}{|l|}{ Classification of epilepsy } \\
\hline \multirow[t]{2}{*}{ Focal } & $13 / 40$ & $3 / 11$ & 1.0 \\
\hline & $32.5 \%$ & $27.3 \%$ & \\
\hline \multirow[t]{2}{*}{ Generalized } & $12 / 40$ & $6 / 11$ & 0.165 \\
\hline & $30.0 \%$ & $54.5 \%$ & \\
\hline \multirow[t]{2}{*}{ Infantile spasms } & $13 / 40$ & $3 / 11$ & 1.0 \\
\hline & $32.5 \%$ & $27.3 \%$ & \\
\hline \multicolumn{4}{|l|}{ Specific semiologies } \\
\hline \multirow[t]{2}{*}{ Absence } & $2 / 40$ & $0 / 11$ & 1.0 \\
\hline & $5.0 \%$ & $0.0 \%$ & \\
\hline \multirow[t]{2}{*}{ Tonic } & $4 / 40$ & $2 / 11$ & 0.598 \\
\hline & $10.0 \%$ & $18.2 \%$ & \\
\hline \multirow[t]{2}{*}{ Clonic } & $2 / 40$ & $0 / 11$ & 1.0 \\
\hline & $5.0 \%$ & $0.0 \%$ & \\
\hline \multirow[t]{2}{*}{ Tonic-clonic } & $8 / 40$ & $2 / 11$ & 1.0 \\
\hline & $20.0 \%$ & $18.2 \%$ & \\
\hline \multirow[t]{2}{*}{ Myoclonic } & $5 / 40$ & $0 / 11$ & 0.572 \\
\hline & $12.5 \%$ & $0.0 \%$ & \\
\hline \multirow[t]{2}{*}{ Other } & $4 / 40$ & $2 / 11$ & 0.598 \\
\hline & $10.0 \%$ & $18.2 \%$ & \\
\hline \multirow[t]{2}{*}{ Epilepsy outcome } & $54 / 82$ & $17 / 31$ & 0.286 \\
\hline & $65.9 \%$ & $54.8 \%$ & \\
\hline \multirow[t]{2}{*}{ Intractable/refractory seizures } & $22 / 34$ & $3 / 8$ & 0.235 \\
\hline & $64.7 \%$ & $37.5 \%$ & \\
\hline \multirow[t]{2}{*}{ Occasional/controlled seizures } & $12 / 34$ & $5 / 8$ & 0.235 \\
\hline & $35.3 \%$ & $62.5 \%$ & \\
\hline \multirow[t]{2}{*}{ Not assessed } & $20 / 54$ & $9 / 17$ & - \\
\hline & $37.0 \%$ & $52.9 \%$ & \\
\hline
\end{tabular}

Prevalence and course of epilepsy, seizures types, and outcome are listed. Proportions are illustrated in numbers (top) and percentages (bottom).

\section{Neuroanatomical and histopathological features}

A detailed description of cortical and extracortical malformations sorted by subgroups is presented in Fig. 3, Tables S5, S6, and Figs. S3, S4.

Cortical malformations were reported in $89.7 \%$ and $97.8 \%$ of individuals in the TUBA1A and TUBB2B cohort. They were present in all deceased individuals and fetuses. Lissencephaly $(32.8 \%$ vs. $6.8 \%)$ with pachygyria and/or agyria was a distinctive cortical finding in TUBA1A compared with TUBB2B tubulinopathy $(P<0.001$; Fig. 3 ; Table S5). Microlissencephaly as the most severe form of the lissencephaly spectrum was more prevalent in the TUBA1A cohort and was reported in $50.0 \%$ of fetal cases whereas only one fetal case was described in the TUBB2B cohort. Simplified gyral pattern as milder form of the spectrum was exclusively described in living individuals and evenly distributed (Fig. 3; Table S5). Polymicrogyria (PMG) and polymicrogyria-like cortical dysplasia (PMG-like CD) were significantly more frequent in individuals of the TUBB2B cohort $(P=0.001$ and $P<0.001$, respectively; Fig. 3; Table $S 5$ ). Extracortical findings were described in all but one case in the $T U B B 2 B$ cohort. Complete cerebellar agenesis, basal ganglia hypertrophy, and brainstem dysplasia were extracortical characteristics exclusively described in the TUBA1A cohort. Additionally, abnormalities of corpus callosum (CC) were significantly more common in TUBA1A tubulinopathy $(96.1 \%$ vs. $77.5 \% ; P<0.001)$. In return, $T U B B 2 B$ tubulinopathy showed significant differences concerning the occurrence of basal ganglia (63.6\% vs. $84.6 \%$; $P=0.02)$ and ventricular dysgenesis, especially ventriculomegaly $(44.3 \%$ vs. $88.0 \% ; P<0.001)$.

Consistent with the distribution pattern, statistical frequency analysis identified neuroradiological features that were significantly associated with a mild or severe course of disease and epilepsy. For example, microlissencephaly, agyria, cerebellar hypoplasia, complete agenesis of the CC, basal ganglia, and olfactory bulb, hippocampal dysplasia, and pons hypoplasia were more common in individuals of the TUBA1A cohort defined as severely affected (i.e., deceased or fetal cases) whereas pachygyria was more frequently found in living individuals. In the $T U B B 2 B$ cohort, agenesis of the olfactory bulb was significantly more prevalent in severely affected individuals while multifocal PMG, PMG-like CD, and ventriculomegaly were associated with a rather milder course of disease (Fig. S3). In the TUBA1A cohort, epilepsy was associated with lissencephaly and agyria. In the $T U B B 2 B$ cohort, epilepsy was associated with complete agenesis of the CC and brainstem hypoplasia (Fig. S4). Incidence of epilepsy and intractable seizures did not significantly differ in deceased individuals.

Histopathological data from clinical reports of fetal cases are summarized in Table S7. Most common findings in fetuses of the TUBA1A cohort were heterotopic neurons (86.4\%), hypoplastic olivary nuclei $(65.0 \%)$, and a disorganized corticospinal tractum $(60.0 \%)$. In the TUBB2B cohort, heterotopic neurons (83.3\%), enlarged germinal zones $(66.7 \%)$, and neuroglial overmigration (50.0\%) were described, with neuroglial overmigration being a distinctive histopathological feature in TUBB2B tubulinopathy $(9.5 \%$ vs. $50.0 \%$; $P=0.024)$.

\section{DISCUSSION}

We quantitatively and comparatively delineate the natural history of TUBA1A and TUBB2B tubulinopathies by assessment of a large meta-cohort of 203 patients and provide their distinctive clinical features. In particular, we define hard clinical endpoints and seminal features at disease onset and during the course of disease as well as distinctive characteristics: TUBA1A tubulinopathy is initially characterized by primary, progressive microcephaly and facial dysmorphisms including retrognathism and hypertelorism, developing facial diplegia and abnormalities of the muscular tone in the course of disease resulting in severe motor impairment. Neuroradiological features primarily include lissencephaly and abnormalities of the corpus callosum. In comparison, patients with $T U B B 2 B$ tubulinopathy show developmental delay at disease onset and are characterized by isolated learning difficulties and congenital fibrosis of the extraocular muscles. 

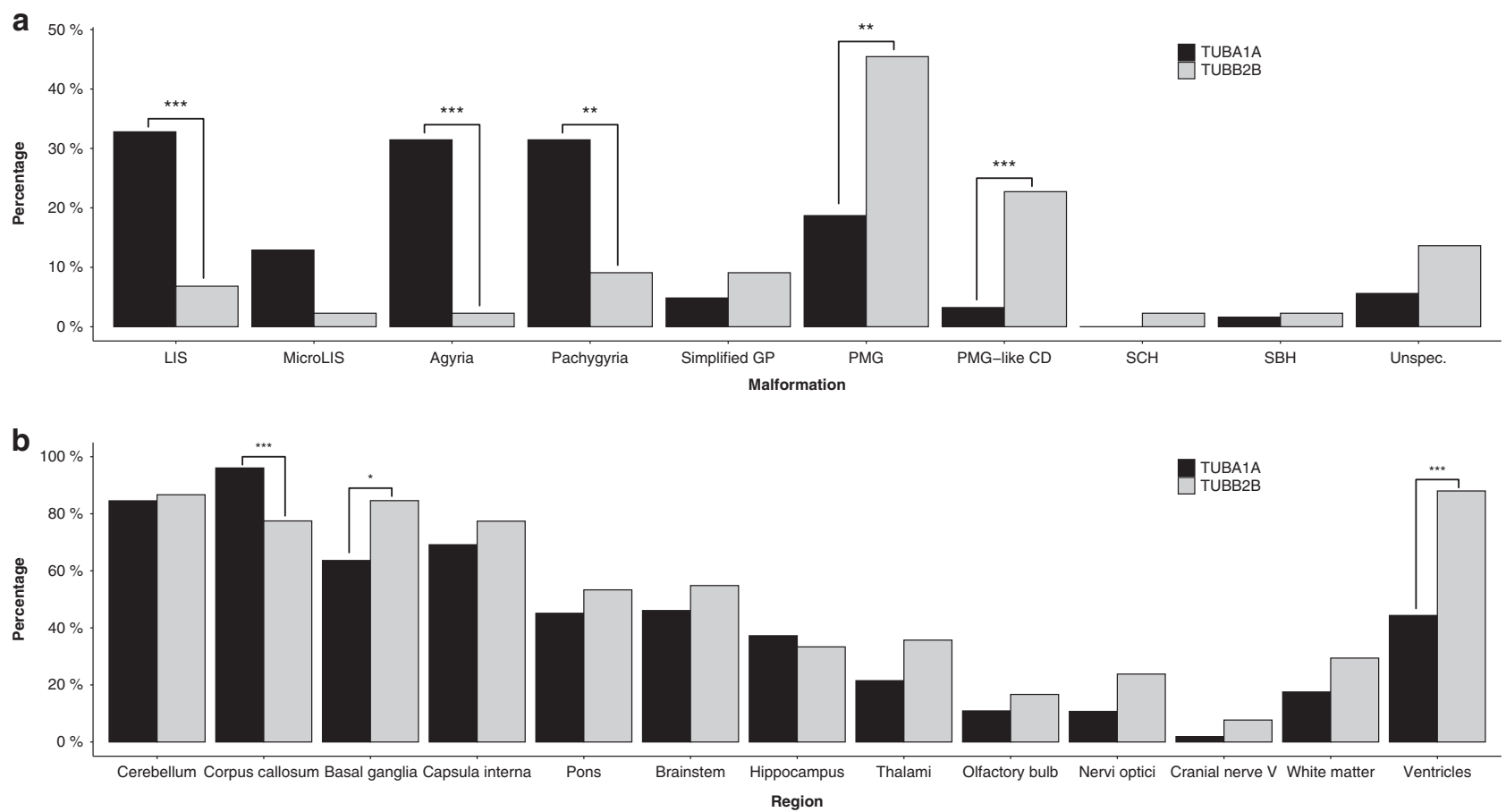

Fig. 3 Frequency of cortical (a) and extracortical findings (b) in the TUBA1A (black) and TUBB2B (gray) cohorts. Statistically significant differences are marked with asterisks. LIS lissencephaly, MicroLIS microlissencephaly, PMG polymicrogyria, PMG-like CD polymicrogyria-like cortical dysplasia, SBH subcortical band heterotopia, SCH schizencephaly, Simplifed GP simplified gyral pattern, Unspec. unspecified. Level of significance: * $\leq 0.05, * * \leq 0.01, * * * \leq 0.001$.

PMG and PMG-like CD, with their microscopic correlate of neuronal overmigration, and abnormalities of the basal ganglia and ventricles are predominant neuroradiological and histopathological features.

Both tubulinopathies showed a relevant estimated mortality of $7 \%$ during the first decade of life. Documented patients in the TUBA1A cohort died earlier, mainly within this vulnerable period. Even though documentation in the literature is sparse, our present data indicate respiratory complications as a main cause of death, likely being the result of severe motor impairment that was present in all deceased patients. This is in line with data from population-based studies that associated neurological impairment to premature death in children. ${ }^{32}$ Mortality of TUBA1A and TUBB2B tubulinopathy might be even higher as fetuses from terminated pregnancies represent a relevant percentage of reported TUBA1A (16.7\%) and TUBB2B (14.9\%) cases.

TUBA1A and TUBB2B tubulinopathies usually become symptomatic in infancy at an average age of 4 and 6 months, respectively, but a considerable diagnostic delay of 4.2 and 12.3 years is observed until a genetic diagnosis is established. Especially individuals with $T U B B 2 B$ tubulinopathy were diagnosed late in childhood. Novel genetic screening tests together with experienced clinicians will likely help to establish an earlier diagnosis for these ultraorphan conditions with distinct central nervous system (CNS) features but unspecific clinical signs. ${ }^{15,33,34}$ In both tubulinopathies presented here, seizures and muscular hypotonia were cardinal symptoms at disease onset. Distinctive features included primary, progressive microcephaly and facial dysmorphisms in individuals with TUBA1A tubulinopathy. Our clinical data suggest a generally milder course of TUBB2B compared with TUBA1A tubulinopathy regarding the time course of estimated survival and the less frequent occurrence of neurological comorbidities such as motor impairment (97.3\% vs. $73.1 \%$ ), abnormalities of the muscular tone $(89.6 \%$ vs. $48.0 \%$ ), and global developmental delay ( $95.7 \%$ vs. $76.7 \%)$. Additionally, nearly $20 \%$ of individuals with TUBB2B tubulinopathy seem to have normal motor function, which is in line with previous reports of a low prevalence of muscular tone abnormalities in TUBB2B tubulinopathy. ${ }^{4}$ Diagnostic delay in TUBB2B tubulinopathy could be partially explained by the more subtle clinical signs at disease onset. According to the literature and derived from our data, patients with TUBA1A compared with TUBB2B tubulinopathy more frequently show severe MCDs such as microlissencephaly, lissencephaly, and agyria. ${ }^{4,16}$ Comparatively, less severe MCDs such as PMG and PMG-like CD, which are also seen in connatal infections, appear more specific for TUBB2B tubulinopathy, therefore not prompting early genetic testing. ${ }^{35,36}$

Epilepsy is a cardinal feature at disease onset of TUBA1A and TUBB2B tubulinopathy: $65.9 \%$ and $54.8 \%$ of patients, respectively, developed seizures during the observation period. In the majority of cases, epilepsy manifests in the first year of life with infantile spasms as the most common epilepsy syndrome. However, the spectrum of semiology and severity is broad and course of epilepsy seems to be more severe in TUBA1A tubulinopathy where epilepsy remained refractive in two thirds of the reported cases. We could show 
that particular malformations of cortical (lissencephaly, agyria) and extracortical (complete agenesis of the CC, brainstem hypoplasia) brain structures were associated with epilepsy in TUBA1A and TUBB2B tubulinopathies, respectively. Accordingly, previous studies suggested basal ganglia and cerebellar dysfunction favoring epileptogenesis. ${ }^{37,38}$ While some authors described the course of epilepsy in tubulinopathies as rather benign, this discrepancy to our study results is likely explained by the different cohort size. ${ }^{20}$

\section{Study limitations and future directions}

Quantitative natural history modeling based on clinical reports is inherently accompanied by several relevant limitations. First, ascertainment bias affects soft variables such as clinical and neuroradiological features. In case of TUBA1A tubulinopathy, a previous study showed clustering of descriptive terms depending on the clinical report from which they originated. ${ }^{5}$ Furthermore, predominance of patients' countries of origin could be biased by large studies executed in developed countries with sufficient resources for comprehensive genetic testing. Second, lack of a standardized study protocol leads to data loss, which is why prospective studies are more suitable for assessment of descriptive variables. Accordingly, this study focuses on hard clinical endpoints such as survival, disease onset, and diagnostic delay. Third, survival estimations are based upon small sample sizes without consideration of possible confounders such as supportive care measures. As tubulinopathies are extremely rare diseases, prospective assessment of survival data would require an international multicenter study approach with a high logistic and temporal burden. In contrast, hard clinical endpoints are provided by quantitative natural history modeling methodology in a timely manner. ${ }^{29}$

\section{Conclusions}

In this present study, we define the important primary endpoints survival, disease onset, and diagnostic delay analyzing the so-far largest quantitative analysis cohort of patients with TUBA1A and TUBB2B tubulinopathy. Thereby, we provide clinical and epidemiological data that are of particular interest for future diagnostic and therapeutic trials. Moreover, we sharpen the phenotypic profile of TUBA1A and $T U B B 2 B$ tubulinopathy by comprehensively delineating isotype-dependent characteristics at disease onset and in the course of disease as well as neuroradiological features associated with epilepsy and an unfavorable clinical outcome. By outlining excessive diagnostic delay of tubulinopathies, we intend to raise awareness of these ultraorphan diseases as diagnostic uncertainty and a relevant proportion of TOPs can cause high psychosocial burden for afflicted families. Earlier diagnosis, e.g., using targeted panel testing, exome sequencing, or prospectively noninvasive prenatal genetic testing following detection of fetal brain abnormalities, is pivotal to counsel parents concerning accurate prognosis and recurrence risk. ${ }^{39}$

\section{SUPPLEMENTARY INFORMATION}

The online version of this article (https://doi.org/10.1038/s41436020-01001-z) contains supplementary material, which is available to authorized users.

\section{ACKNOWLEDGEMENTS}

This study makes use of data generated by the DECIPHER community. A full list of centers that contributed to the generation of the data is available from https://decipher.sanger. ac.uk and via email from decipher@sanger.ac.uk. Funding for the DECIPHER project was provided by Wellcome. We especially thank the DECIPHER contributors E. Bijlsma, A. van Haeringen, and C.A.L. Ruivenkamp (University Hospital Leiden, The Netherlands) for data collection and coordination. This study was supported by grants from the Dietmar Hopp Foundation (grant 1DH1813319 to SS).

\section{FUNDING}

Open Access funding enabled and organized by Projekt DEAL.

\section{DISCLOSURE}

The authors declare no conflicts of interest.

Publisher's note Springer Nature remains neutral with regard to jurisdictional claims in published maps and institutional affiliations.

\section{REFERENCES}

1. Ayala R, Shu T, Tsai LH. Trekking across the brain: the journey of neuronal migration. Cell. 2007;128:29-43.

2. Tischfield MA, Engle EC. Distinct alpha- and beta-tubulin isotypes are required for the positioning, differentiation and survival of neurons: new support for the 'multi-tubulin' hypothesis. Biosci Rep. 2010;30:319-330.

3. Minoura I. Towards an understanding of the isotype-specific functions of tubulin in neurons: Technical advances in tubulin expression and purification. Neurosci Res. 2017;122:1-8.

4. Bahi-Buisson N, Poirier K, Fourniol F, et al. The wide spectrum of tubulinopathies: what are the key features for the diagnosis? Brain. 2014;137:1676-1700.

5. Hebebrand $M$, Huffmeier $U$, Trollmann $R$, et al. The mutational and phenotypic spectrum of TUBA1A-associated tubulinopathy. Orphanet J Rare Dis. 2019;14:38.

6. Keays DA, Tian G, Poirier K, et al. Mutations in alpha-tubulin cause abnormal neuronal migration in mice and lissencephaly in humans. Cell. 2007; 128:45-57.

7. Poirier K, Lebrun N, Broix L, et al. Mutations in TUBG1, DYNC1H1, KIF5C and KIF2A cause malformations of cortical development and microcephaly. Nat Genet. 2013;45:639-647.

8. Poirier K, Saillour Y, Bahi-Buisson N, et al. Mutations in the neuronal sstubulin subunit TUBB3 result in malformation of cortical development and neuronal migration defects. Hum Mol Genet. 2010;19:4462-4473.

9. Breuss $M$, Heng Jl, Poirier $K$, et al. Mutations in the beta-tubulin gene TUBB5 cause microcephaly with structural brain abnormalities. Cell Rep. 2012;2:1554-1562.

10. Abdollahi MR, Morrison E, Sirey $T$, et al. Mutation of the variant alphatubulin TUBA8 results in polymicrogyria with optic nerve hypoplasia. Am J Hum Genet. 2009;85:737-744.

11. Cushion TD, Paciorkowski AR, Pilz DT, et al. De novo mutations in the beta-tubulin gene TUBB2A cause simplified gyral patterning and infantile-onset epilepsy. Am J Hum Genet. 2014;94:634-641.

12. Jaglin $X H$, Poirier $K$, Saillour $Y$, et al. Mutations in the beta-tubulin gene TUBB2B result in asymmetrical polymicrogyria. Nat Genet. 2009:41:746-752.

13. Lohmann K, Wilcox RA, Winkler S, et al. Whispering dysphonia (DYT4 dystonia) is caused by a mutation in the TUBB4 gene. Ann Neurol. 2013;73:537-545. 
14. Cushion TD, Dobyns WB, Mullins JG, et al. Overlapping cortical malformations and mutations in TUBB2B and TUBA1A. Brain. 2013; 136:536-548.

15. Di Donato N, Timms AE, Aldinger KA, et al. Analysis of 17 genes detects mutations in $81 \%$ of 811 patients with lissencephaly. Genet Med. 2018;20:1354-1364.

16. Romaniello R, Arrigoni F, Fry AE, et al. Tubulin genes and malformations of cortical development. Eur J Med Genet. 2018;61:744-754.

17. Cederquist GY, Luchniak A, Tischfield MA, et al. An inherited TUBB2B mutation alters a kinesin-binding site and causes polymicrogyria, CFEOM and axon dysinnervation. Hum Mol Genet. 2012;21: 5484-5499.

18. Tischfield MA, Baris $H N$, Wu C, et al. Human TUBB3 mutations perturb microtubule dynamics, kinesin interactions, and axon guidance. Cell. 2010;140:74-87.

19. Romaniello R, Arrigoni F, Panzeri E, et al. Tubulin-related cerebellar dysplasia: definition of a distinct pattern of cerebellar malformation. Eur Radiol. 2017;27:5080-5092.

20. Romaniello R, Zucca C, Arrigoni F, et al. Epilepsy in tubulinopathy: personal series and literature review. Cells. 2019;8:669.

21. Orphanet Report Series. Prevalence of rare diseases: bibliographic data. Number 1. January 2020. http://www.orpha.net/orphacom/cahiers/docs/ GB/Prevalence_of_rare_diseases_by_alphabetical_list.pdf. Accessed 24 February 2020.

22. Garbade SF, Zielonka M, Komatzsuzaki S, et al. Quantitative retrospective natural history modeling for orphan drug development. J Inherit Metab Dis. 2020. https://doi.org/10.1002/jimd.12304. [Epub ahead of print].

23. von Elm E, Altman DG, Egger $M$, et al. The Strengthening the Reporting of Observational Studies in Epidemiology (STROBE) statement: guidelines for reporting observational studies. Lancet. 2007;370:1453-1457.

24. Landrum MJ, Lee JM, Benson M, et al. ClinVar: improving access to variant interpretations and supporting evidence. Nucleic Acids Res. 2018:46:D1062-D1067.

25. Firth HV, Richards SM, Bevan AP, et al. DECIPHER: Database of Chromosomal Imbalance and Phenotype in Humans Using Ensembl Resources. Am J Hum Genet. 2009;84:524-533.

26. Wildeman M, van Ophuizen E, den Dunnen JT, Taschner PE. Improving sequence variant descriptions in mutation databases and literature using the Mutalyzer sequence variation nomenclature checker. Hum Mutat. 2008;29:6-13.

27. Palisano RJ, Rosenbaum P, Bartlett D, Livingston MH. Content validity of the expanded and revised Gross Motor Function Classification System. Dev Med Child Neurol. 2008;50:744-750.

28. Komatsuzaki S, Zielonka M, Mountford WK, et al. Clinical characteristics of 248 patients with Krabbe disease: quantitative natural history modeling based on published cases. Genet Med. 2019;21:2208-2215.
29. Zielonka M, Garbade SF, Kolker S, Hoffmann GF, Ries M. A crosssectional quantitative analysis of the natural history of free sialic acid storage disease-an ultraorphan multisystemic lysosomal storage disorder. Genet Med. 2019;21:347-352.

30. R Core Team. R: A language and environment for statistical computing. $\mathrm{R}$ Foundation for Statistical Computing, Vienna, Austria. 2013. http://www.R-project.org/.

31. Kahle D, Wickham H. ggmap: spatial visualization with ggplot2. R J. 2013;5:144-161.

32. Decoufle $P$, Autry A. Increased mortality in children and adolescents with developmental disabilities. Paediatr Perinat Epidemiol. 2002;16:375-382.

33. Parrini E, Conti V, Dobyns WB, Guerrini R. Genetic basis of brain malformations. Mol Syndromol. 2016;7:220-233.

34. Lemke JR, Riesch E, Scheurenbrand T, et al. Targeted next generation sequencing as a diagnostic tool in epileptic disorders. Epilepsia. 2012; 53:1387-1398.

35. Van den Veyver IB. Prenatally diagnosed developmental abnormalities of the central nervous system and genetic syndromes: a practical review. Prenat Diagn. 2019;39:666-678.

36. Cabet S, Karl K, Garel C, et al. Two different prenatal imaging cerebral patterns of tubulinopathies. Ultrasound Obstet Gynecol. 2020. https://doi. org/10.1002/uog.22010. [Epub ahead of print].

37. Blumenfeld H, Varghese GI, Purcaro MJ, et al. Cortical and subcortical networks in human secondarily generalized tonic-clonic seizures. Brain. 2009;132:999-1012.

38. Rektor I, Tomcik J, Mikl M, Marecek R, Brazdil M, Rektorova I. Association between the basal ganglia and large-scale brain networks in epilepsy. Brain Topogr. 2013;26:355-362.

39. Zhang J, Li J, Saucier JB, et al. Non-invasive prenatal sequencing for multiple Mendelian monogenic disorders using circulating cell-free fetal DNA. Nat Med. 2019;25:439-447.

\section{cc) (i)}

Open Access This article is licensed under a Creative Commons Attribution 4.0 International License, which permits use, sharing, adaptation, distribution and reproduction in any medium or format, as long as you give appropriate credit to the original author(s) and the source, provide a link to the Creative Commons license, and indicate if changes were made. The images or other third party material in this article are included in the article's Creative Commons license, unless indicated otherwise in a credit line to the material. If material is not included in the article's Creative Commons license and your intended use is not permitted by statutory regulation or exceeds the permitted use, you will need to obtain permission directly from the copyright holder. To view a copy of this license, visit http://creativecommons.org/licenses/ by/4.0/.

(C) The Author(s) 2020 